\title{
Band structures and transport properties of zigzag graphene nanoribbons with antidot arrays
}

\author{
Ying-Tao Zhang, Qing-Ming Li, and You-Cheng Li \\ College of Physics, Hebei Normal University, Shijiazhuang 050016, China \\ Yan-Yang Zhang \\ Department of Physics, The University of Hong Kong, Pokfulam Road, Hong Kong \\ Feng Zhai \\ School of Physics and Optoelectronic Technology, College of Advanced Science and Technology, \\ Dalian University of Technology, Dalian 116024, China
}

(Dated: January 31, 2011)

\begin{abstract}
We present a study on the band and transport features of a zigzag graphene nanoribbon with an antidot lattice. It is found that the inclusion of an infinite antidot lattice turns the original semimetal zigzag-edged graphene nanoribbon to a gapped semiconductor. The size of the band gap can be tuned by the position of the antidots and the distance $D$ between two nearest antidots and depends on the ribbon width. For a finite superlattice with $N$ antidots and a large $D$, a group of $(N-1)$ splitting resonant peaks and transmission-blockade regions appear alternatively in the conductance spectrum. This indicates the formation of minibands and minigaps. In addition, Fano resonances can be observed when the antidots are localized near one edge of the nanoribbon. These features provide potential applications for graphene-based electronic and optoelectronic devices.
\end{abstract}

PACS numbers: 73.63.-b, 73.23.-b, 73.20.At, 73.20.Hb

\section{INTRODUCTION}

Graphene, a monolayer of carbon atoms with a twodimensional honeycomb lattice, has become one of the most promising new super-materials in nanoelectronics since its successful fabrication in 2004[1-7]. The unconventional properties of graphene materials have inspired lots of potential applications. For example, chemical sensors capable of detecting individual gas molecules have been demonstrated based on the high sensitivity of graphene to molecular doping from gaseous molecules[8]. Due to the high electrical conductivity and optical transparency, graphene is made as candidate for transparent conducting electrodes and leads in solar cells[9] and touch screen displays. However, bulk graphene is a gapless material, which limits the on-off ratios of graphenebased transistors.

The antidot superlattice[10], which is an array of insulating islands in a two-dimensional electron gas, is a wellstudied class of man-made structures due to their interesting transport properties related to the formation of miniband and minigap structure and quantum interference. The periodic antidot lattice in graphene is typically achieved by removing carbon atoms by means of electron-beam lithography and etching techniques. Since Pedersen et al.[11, 12] demonstrated that the antidot lattices could turn the semimetallic bulk graphene into a gapped semiconductor, the property of antidots in graphene has attracted great interests for potential applications in electronics and optoelectronics. Vanević et al.[13] utilized a $\pi$-orbital tight-binding model to study graphene nanostructure with rather large triangular antidot lattices and predicted the appearance of flat bands and the formation of spatial localization. Rosales et al.[14] studied the transport properties of antidot superlattices on armchair graphene nanoribbons and presented the relation between the gap structure and the period of antidot lattices on the nanorib- bon. In addition, a new class of massless Dirac fermions have been predicted in graphene when a periodic potential is applied, for which the wave vector and group velocity are not collinear[15, 16]. In experiment, Eroms et al.[17] have fabricated and measured antidot lattices in single-layered graphene with a lattice period down to $90 \mathrm{~nm}$, and observed a transport gap of a few $\mathrm{mV}$ around the Dirac point. Another experimental realization of the antidot array in graphene has been presented by Shen et al., who fabricated square antidot arrays on graphene films and observed Aharonov-Bohm oscillations arising from modulation of this artificial potential[18]. Very recently, individual vacancies of a diameter $\approx 1 \AA$ have been realized in carbon nanotubes[19]. These technologies have paved the way for the realization of graphene antidot lattices with arbitrary geometries.

It is well known that the graphene nanoribbon with a zigzag edges differs greatly from that with armchair edges. It is thus interesting to explore the band and transport features of zigzag graphene nanoribbons with an antidot superlattice. In this work, we present such a study. We find that the conductance show Fano-like resonances when the antidots are close to one of the two zigzag edges. The band gap caused by the antidot lattice can be tuned continuously by changing the position of the antidots. For a finite antidot superlattice with $N$ unit cells, $(N-1)$ conductance peaks appear in the miniband of the corresponding perfect antidot superlattice. These properties would be interesting for electronic and optoelectronic applications.

The remainder of the paper is organized as follows. In Sec. II, the model and calculation methods are described. The numerical results and discussions are presented in Sec. III. Finally, we summarize our findings in Sec. IV. 


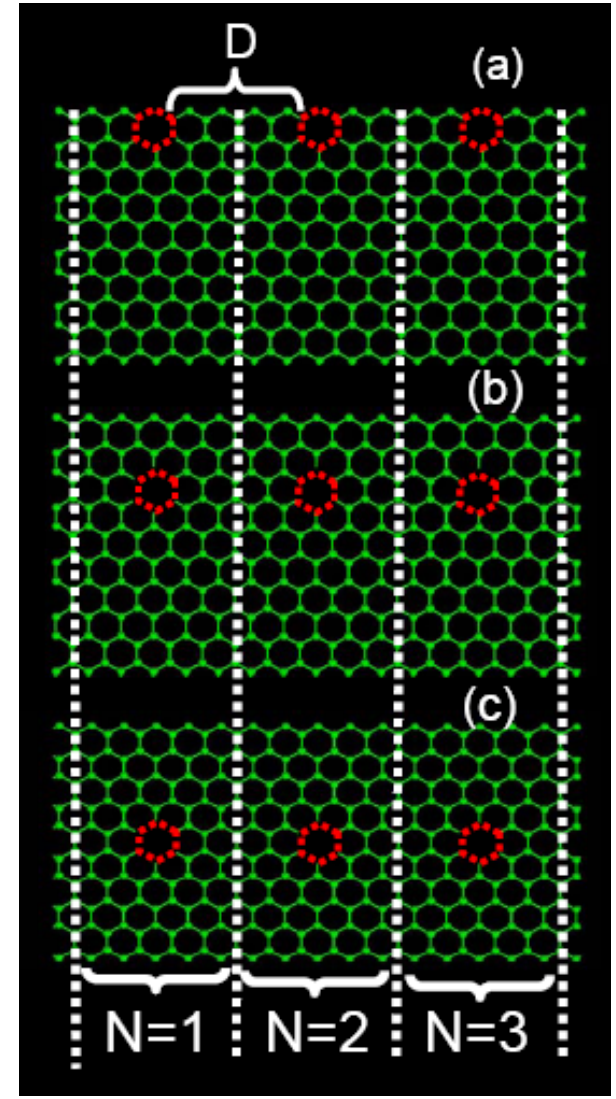

FIG. 1: (Color online) Illustration of a zigzag graphene nanoribbon with an antidot lattice. A hexagon-shaped antidot defect forms when all of the six atoms in a hexagon are removed. (a) The antidots are near one edge of the nanoribbon. (b) The antidots locate in a general position of the nanoribbon. (c) The antidots locate in the middle of the nanoribbon. The white dashed lines mark the interfaces between two unit cells.

\section{MODEL AND METHOD}

We consider a zigzag graphene nanoribbon made up of $N_{C}$ carbon chains. Some of the carbon atoms in the nanoribbon sample are removed to form an antidot superlattice with $N$ unit cells. Two semi-infinite zigzag graphene leads are attached to the two terminals of the sample. The motion of $\pi$ electrons in such a nanoribbon with periodic defects can be described by the following tight-binding model:

$$
H=-\sum_{i, j} t_{i j}\left(c_{i}^{\dagger} c_{j}+\text { H.c. }\right)
$$

where $c_{i}^{\dagger}\left(c_{i}\right)$ creates (annihilates) an electron in the $\pi$ orbital located at site $i$, and the hopping integral $t_{i j}$ is $t \approx 3 \mathrm{eV}$ when the site $i$ is in the nearest neighborhood with the site $j$ and zero otherwise. For concreteness, an hexagon-type antidot with six extracted atoms is considered. The separation between two adjacent antidots is set to be $D$ in unit of the lattice constant $a$ of bulk graphene. Thus the period length of the antidot superlattice is $L=(D+1) a$. As an illustrative example, Fig. 1 shows a $N_{C}=10$ zigzag nanoribbon with

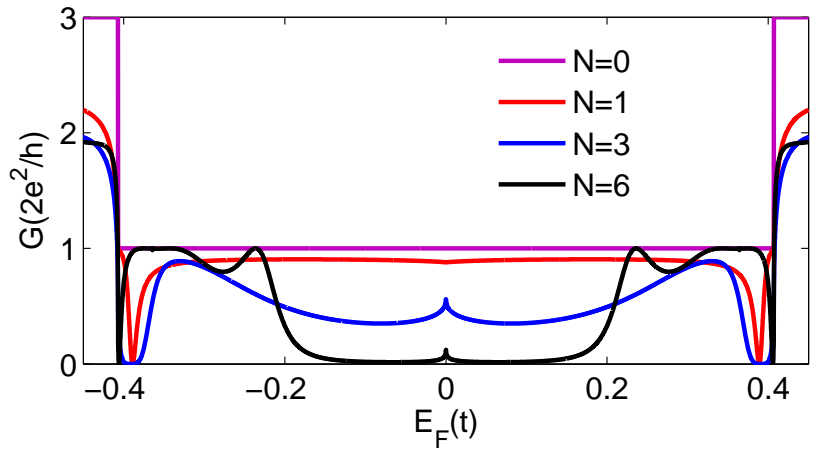

FIG. 2: (Color online) The conductance as a function of the Fermi energy for the $N_{C}=10$ zigzag graphene nanoribbon with one or several antidots near one edge. The number of antidots is $N=1$ (red line), 3 (blue line), and 6 (black line). The distance between two nearest antidots is $4 a$. The conductance of a perfect zigzag graphene nanoribbon is plotted (the magenta line) as reference.

$N=3$ antidots. The antidot can be placed in different positions, such as the edge [Fig. 1(a)], the middle [Fig. 1(c)] and other part [Fig. 1(b)] of the nanoribbon. It should be pointed out that the shape of the antidots plays a minor role in the band and transport features discussed below.

At zero temperature and for a given Fermi energy $E_{F}$, the two-terminal conductance of the considered system can be calculated by the Landauer formula[20]

$$
G\left(E_{F}\right)=\frac{2 e^{2}}{h} \operatorname{Tr}\left[\Gamma_{S}\left(E_{F}\right) \mathbf{G}^{\mathbf{r}}\left(E_{F}\right) \Gamma_{D}\left(E_{F}\right) \mathbf{G}^{\mathbf{a}}\left(E_{F}\right)\right],
$$

where $\mathbf{G}^{\mathbf{r}(\mathbf{a})}\left(E_{F}\right)=\left[E_{F} I-H_{c e n}-\Sigma_{S}^{r(a)}(E)-\Sigma_{D}^{r(a)}(E)\right]^{-1}$ is the retarded (advanced) Green function of the central region, $\Gamma_{S(D)}\left(E_{F}\right)=i\left\{\Sigma_{S(D)}^{r}\left(E_{F}\right)-\left[\Sigma_{S(D)}^{r}\left(E_{F}\right)\right]^{\dagger}\right\}$ is the line-width function, and $\Sigma_{S(D)}^{r}\left(E_{F}\right)$ is the self energy due to the semi-infinite source (drain), which can be calculated numerically in a recursive way.[21-23]

\section{NUMERICAL RESULTS}

In Fig. 2, we show the conductance of a zigzag graphene nanoribbon with antidot defects as a function of the Fermi energy. The hexagon-type antidots are near one edge of the nanoribbon [see Fig. 1(a)]. For a perfect zigzag nanoribbon, quantized conductance plateau shows up. The presence of one or several antidots near one edge changes the boundary condition of electrons in the nanoribbon. The conductance is thus modified. Actually, Fano resonances appear when the Fermi energy is close to the threshold of the second transport mode. It can be illustrated by the interference between the propagating states and bound states in the zigzag graphene nanoribbon with antidot defect(s). The bound state is induced by the presence of antidot(s) near one edge of the nanoribbon. The edge states near another perfect zigzag edge provide the propagating states. In addition, the width of Fano resonances can be 


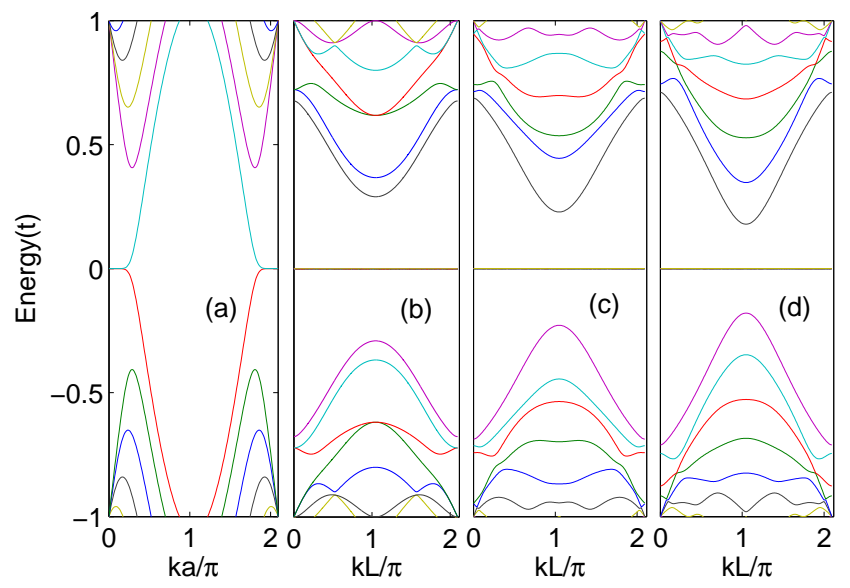

FIG. 3: (Color online) Electronic band structures for the $N_{C}=10$ zigzag graphene nanoribbon with an infinite antidot superlattice. The distance between two nearest antidots is $4 a$. The positions of antidots in (b)-(d) are illustrated in Figs. 1(c), 1(b), and 1(a), respectively. The band structure for the perfect zigzag nanoribbon is shown in (a) for reference.

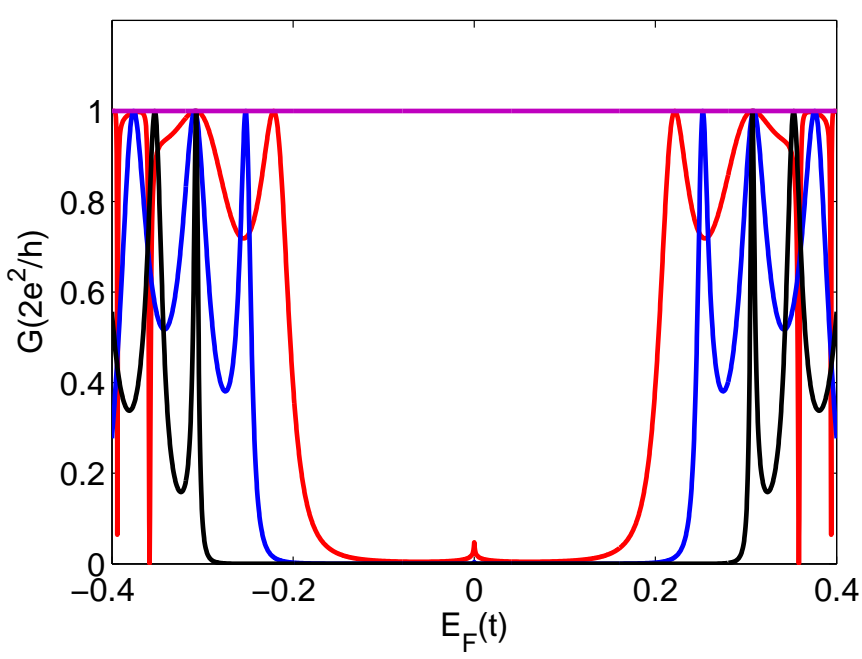

FIG. 4: (Color online) The conductance as a function of the Fermi energy for the $N_{C}=10$ zigzag graphene nanoribbon with 7 antidots near one edge. The distance between two nearest antidots is $4 a$. The red, blue, and black lines are for the positions of antidots illustrated in Figs. 1(a)-1(c), respectively. The magenta line is for the perfect zigzag graphene nanoribbon.

adjusted by the number of antidots $N$. As $N$ increases, the conductance becomes small until the occurrence of a zeroconductance plateau in the single-channel transport regime. This fact indicates the formation of a minigap in the nanoribbon with an antidot superlattice.

Figure 3 shows the band structure of a zigzag graphene nanoribbon with an infinite antidot superlattice. The three different positions of antidots shown in Fig. 1 are considered. Perfect graphene nanoribbons with zigzag edges show an un-

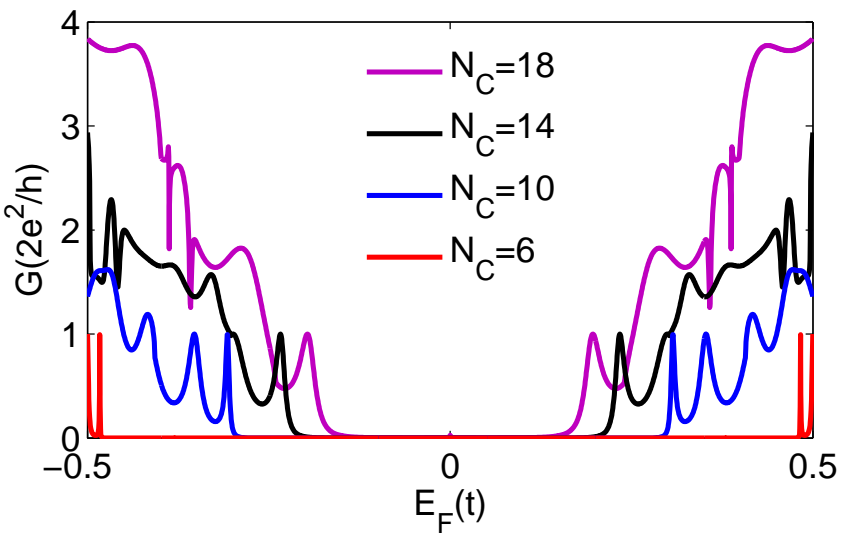

FIG. 5: (Color online) The conductance as a function of the Fermi energy for the zigzag graphene nanoribbon with 7 antidots located in the middle of the nanoribbon. The distance between two nearest antidots is $4 a$. The number of carbon chains is set at $N_{C}=6$ (red line), 10 (blue line), 14 (black line), and 18 (magenta line).

usual semimetallic behavior[25], as shown in Fig. 3(a). The presence of an antidot superlattice results in a band gap [see Figs. 3(b)-3(d)]. Such a transition from a semimetal to a semiconductor has been demonstrated in graphene sheets[11, 12] when regularly spaced holes are introduced. It can be seen from Fig. 3 that the size of the band gap is the largest when the antidots locate in the middle of the nanoribbon. With the position of antidot defects moving from the middle to the edge of the nanoribbon, the size of the band gap decreases gradually. For a finite antidot superlattice, minigaps are formed and within the band gap of the corresponding perfect superlattice, which together with the minibands are the basic features for finite superlattice structures. Some conductance features of a finite antidot superlattice, as shown in Fig. 4, can be understood from this viewpoint. The zero-conductance plateau in the single-channel transport regime arises from the minigap. The ranges of zero-conductance plateau are extended when the antidot positions move from the edge to the middle of the nanoribbon, which is consistent with the features of the band gap in Fig. 3. Beyond the zero-conductance region, the conductance shows an obvious resonance tunneling behavior for the antidot positions shown in Figs. 1(b) and 1(c) due to the presence of miniband states. When the antidots locate in one edge of the nanoribbon, Fano dips appear as observed in Fig. 2.

We have presented that the size of the band gap of an antidot superlattice can be adjusted by changing the position of antidots. The band gap also depends on the width of the nanoribbon characterized by $N_{C}$. In Fig. 5 , the conductance is plotted as a function of the Fermi energy for the antidot superlattice with 7 unit cells and different ribbon width, $N_{C}=6,10,14$, and 18. Hereafter we consider the case that the antidots locate in the middle of the ribbon. However, similar band and transport features discussed below can be observed when the antidots are in other positions such as that in Figs. 1(a) and 1(c). It can be observed that the range of zero-conductance plateau 

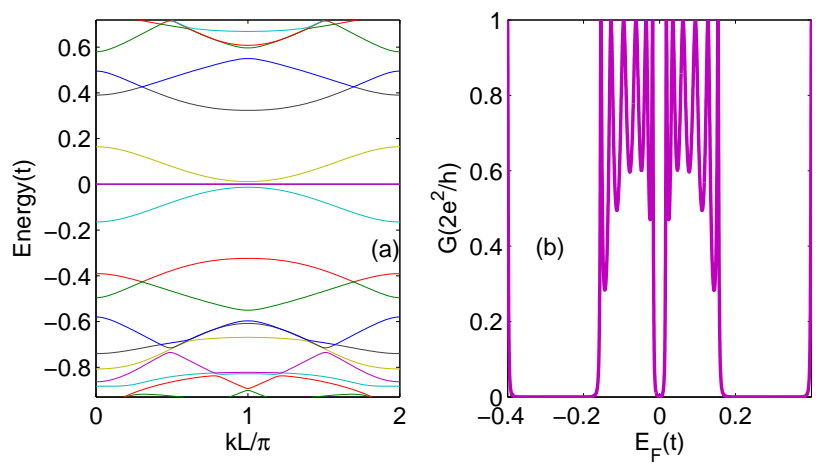

FIG. 6: (Color online) (a) Electronic band structures for the $N_{C}=$ 10 zigzag graphene nanoribbon with an antidot superlattice. The distance between two nearest antidots is $8 a$. The antidots are in the middle of the nanoribbon. (b) The conductance as a function of the Fermi energy for the corresponding finite superlattice with $N=7$.

decreases with the increasing of $N_{C}$, as expected. Besides the resonant tunneling feature, sharp antiresonance peaks can be seen in the multi-mode transport regime.

In our proposed scheme, the minibands and minigaps can be further tuned by the change of the distance between two nearest antidots, as shown in Figs. 6 and 7. The number of unit cells is fixed at $N=7$. The comparison between Figs. 3(a) and 6(a) tells us that a miniband appears inside the original band gap of the perfect superlattice as the distance between two nearest antidots is changed from $4 a$ to $8 a$. For a finite superlattice, the resonance splitting effect can be seen in the conductance curves when the Fermi energy is within the miniband [see Fig. 6(b)]. This can be understood as follows. The stripe of the graphene ribbon including an antidot can be viewed as a barrier for electrons because the antidot pushes the local energy levels away from the Dirac points. The stripe regions between two adjacent stripes with an antidot form the potential wells. For a finite superlattice with $N$ barriers, the number of resonant splittings within each miniband is $(N-1)$ fold.[26] Thus 6 resonant peaks are seen in Fig. 6(b) where the antidot number is $N=7$. As the distance between two nearest antidots is further increased, more minigaps and minibands appear. Accordingly, a group of $(N-1)$-fold splitting resonant peaks and transmission-blockade regions appear alternatively in the conductance spectrum, as shown in Fig. 7. The formation of tunable minibands and minigaps in graphene samples provides potential applications in graphene-based optoelectronic devices.

\section{CONCLUSIONS}

In summary, we have studied the band structures and the transport properties of a zigzag graphene nanoribbon with an antidot superlattice based on a tight-binding model. The presence of an infinite antidot superlattice results in band gaps, which depend strongly on the position of antidots, the distance between two nearest antidots, and the ribbon width. For
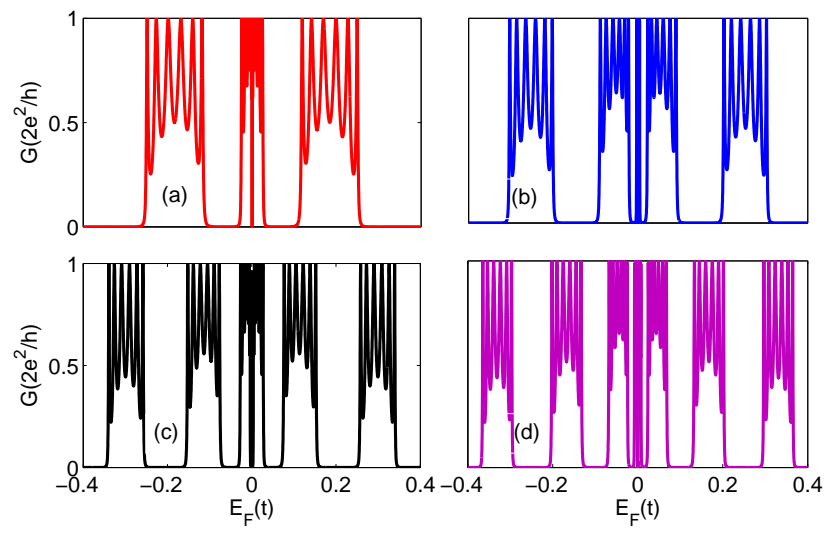

FIG. 7: (Color online) The conductance as a function of the Fermi energy for the zigzag graphene nanoribbon with 7 antidots in the middle of the nanoribbon. The distance between two nearest antidots is (a) $12 a$, (b) $16 a$, (c) $20 a$, and (d) $24 a$.

a finite antidot superlattice, Fano resonances are found when the antidots are localized on one edge of the ribbon. With the increasing of the distance between two nearest antidots, a group of splitting resonant peaks and transmission-forbidden regions appear alternatively in the conductance spectrum. The resonance splitting of the conductance peak is $(N-1)$ fold for the superlattice with $N$ antidots. Such features are related with the formation of minigaps and minibands in the superlattice structures, and could be interesting for electronic and optoelectronic applications.

\section{ACKNOWLEDGMENTS}

We gratefully acknowledge the financial support from NSFC under Grant Nos. 10647126 and 10704013 and Natural Science Foundation of Hebei Province of China under Grant No. A2010000339.
[1] K. S. Novoselov, A. K. Geim, S. V. Morozov, D. Jiang, Y. Zhang, S. V. Dubonos, I. V. Grigorieva, A. A. Firsov, Science 306, 666 (2004).

[2] K. S. Novoselov, A. K. Geim, S. V. Morozov, D. Jiang1, M. I. Katsnelson, I. V. Grigorieva, S. V. Dubonos and A. A. Firsov, Nature 438, 197 (2005).

[3] Yuanbo Zhang, Yan-Wen Tan, Horst L. Stormer and Philip Kim,
Nature 438, 201 (2005).

[4] Geim, A. K. and Novoselov, K. S., Nature Materials 6, 183 (2007).

[5] M. I. Katsnelson, Mater. Today 10, 20 (2007).

[6] A. K. Geim, Science 324, 1530 (2009).

[7] A. H. Castro Neto, F. Guinea, N. M. R. Peres, K. S. Novoselov, and A. K. Geim, Rev. Mod. Phys. textbf81, 109 (2009). 
[8] Schedin, F., A. K. Geim, S. V. Morozov, D. Jiang, E. H. Hill, P. Blake, and K. S. Novoselov, Nature Mater. 6, 652 (2007).

[9] Xuan Wang, Linjie Zhi, and Klaus Müllen, Nano Lett. 8, 323 (2008).

[10] D. Weiss, M. L. Roukes, A. Menschig, P. Grambow, K. von Klitzing, and G. Weimann, Phys. Rev. Lett. 66, 2790 (1991).

[11] T. G. Pedersen, C. Flindt, J. Pedersen, N. A. Mortensen, A.-P. Jauho, and K. Pedersen, Phys. Rev. Lett. 100, 136804 (2008).

[12] T. G. Pedersen, C. Flindt, J. Pedersen, A. P. Jauho, N. A. Mortensen, and K. Pedersen, Phys. Rev. B 77, 245431 (2008).

[13] M. Vanević, V. M. Stojanović, and M. Kindermann, Phys. Rev. B 80, 045410 (2009).

[14] L. Rosales, M. Pacheco, Z. Barticevic, A. León, A. Latgé, and P. A. Orellana, Phys. Rev. B 80, 073402 (2009).

[15] Cheol-Hwan Park, Li Yang, Young-Woo Son, Marvin L. Cohen, and Steven G. Louie, Phys. Rev. Lett. 101, 126804 (2008).

[16] Cheol-Hwan Park, Li Yang, Young-Woo Son, Marvin L. Cohen, Steven G. Louie, Nature Phys. 4, 870 (2008).
[17] J. Eroms and D. Weiss, New J. Phys. 11, 095021 (2009).

[18] T. Shen, Y. Q. Wu, M. A. Capano, L. P. Rokhinson, L. W. Engel, and P. D. Ye, Appl. Phys. Lett. 93, 122102 (2008).

[19] Julio A. Rodriguez-Manzo and Florian Banhart, Nano Lett. 9, 2285 (2009).

[20] S. Datta, Electronic Transport in Mesoscopic Systems (Cambridge University Press, Cambridge, UK, 1995); S. Datta, Quantum Transport: Atom to Transistor (Cambridge University Press, Cambridge, UK, 2005).

[21] D. H. Lee and J. D. Joannopoulos, Phys. Rev. B 23, 4997 (1981).

[22] M. P. Lopez Sancho et al., J. Phys. F 14, 1205 (1984).

[23] M. P. Lopez Sancho et al., J. Phys. F 15, 851 (1985).

[24] U. Fano, Phys. Rev. 124, 1866 (1961).

[25] Wallace, P. R., Phys. Rev. 71, 622 (1947).

[26] G. Bastard, Wave Mechanics Applied to Semiconductor Heterostructures (Les Editions de Physique, Les Ulis, 1988). 